\title{
Insulin resistance is associated with larger thyroid volume in adults with type 1 diabetes
}

\section{Anita Rogowicz-Frontczak, Stanisław Piłaciński, Anna Chwiałkowska, Bogna Wierusz- Wysocka, Dorota Zozulińska- Ziółkiewicz Department of Diabetology and Internal Medicine}

\section{Background:}

Larger thyroid volume and nodular thyroid disease were found to be associated with insulin resistance in patients with type 2 diabetes. Similar analyses is lacking in type 1 diabetes (T1DM).

Aims:

To check the association between thyroid volume and insulin resistance (IR) using estimated glucose disposal rate (eGDR) in patients T1DM.

Materials and methods:

99 consecutive patients with T1DM (44 women, 55 men) aged 29 (mean, SD=6) with diabetes duration 13 (6) years, Exclusion criteria: history of thyroid disease, current treatment of L-thyroxin or anti-thyroid drugs.

Thyroid evaluation:

Anti-thyroid peroxidase (ATPO), anti-thyroglobulin (ATg), anti- thyroid stimulating hormone (TSH) receptor (TRAb) antibodies, assays for TSH and free thyroid hormones (trijodothyronine, fT3, and thyroxin, fT4).

Thyroid was measured using ultrasonography.

Insulin resistance estimation:

eGDR= 24.4 -12.97 x WHR -3.39 x HT - 0.60 x HbA1C

WHR -waist-to-hip ratio, HT - history of hypertension (yes $=1$, no $=0$ ).

IR- if eGDR lower or equal 7.5

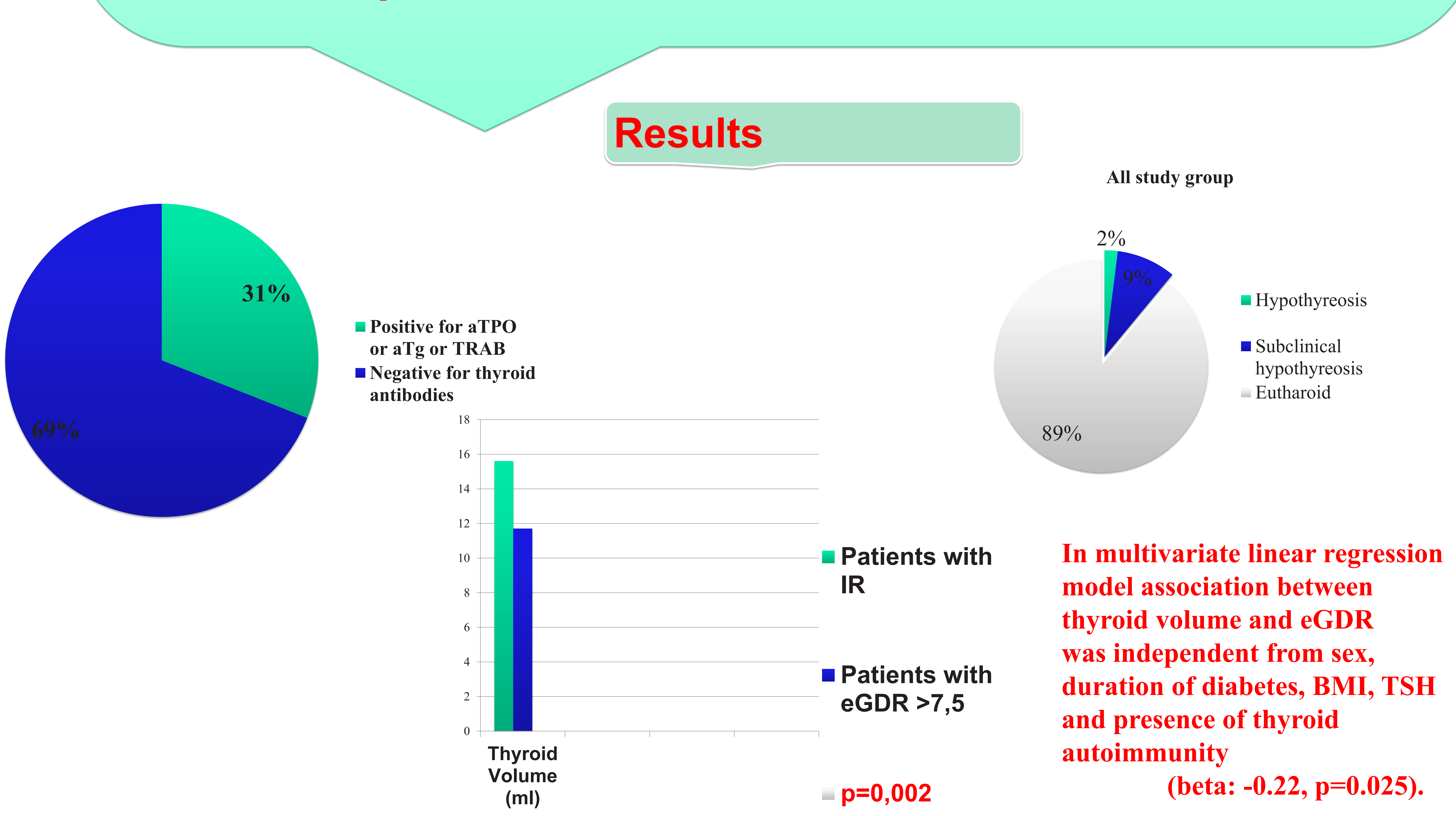

Conclusion: Lower insulin sensitivity is associated with larger thyroid volume in patients with type 1 diabetes independently from body mass index and signs of autoimmune thyroid disease 